\title{
The Baikal Neutrino Project: Present and Perspective
}

V. Aynutdinov ${ }^{\mathrm{a}}$, A. Avrorin ${ }^{\mathrm{a}}$, V. Balkanova, I. Belolaptikov ${ }^{\mathrm{d}}$, D. Bogorodsky ${ }^{\mathrm{b}}$, N. Budnev ${ }^{\mathrm{b}}$, I. Danilchenko ${ }^{\mathrm{a}}$, G. Domogatsky ${ }^{\mathrm{a}}$, A. $^{\mathrm{a}}$

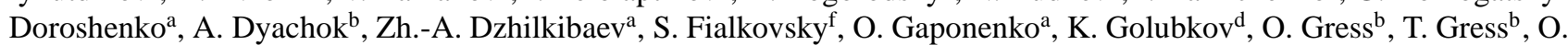
Grishin $^{\text {b }}$, A. Klabukov ${ }^{\mathrm{a}}$, A. Klimov ${ }^{\mathrm{h}}$, A. Kochanov ${ }^{\mathrm{b}}$, K. Konischev ${ }^{\mathrm{d}}$, A.Korobchenko ${ }^{\mathrm{b}}$, A. Koshechkin ${ }^{\mathrm{a}}$, V. Kulepov ${ }^{\mathrm{f}}$, D.

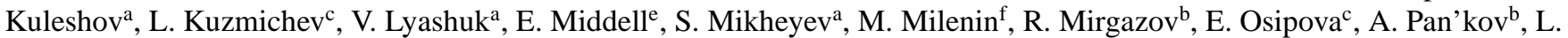

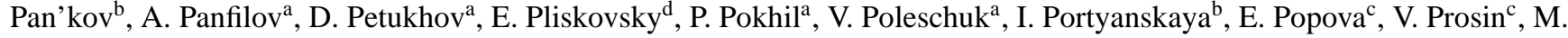
Rozanov $^{g}$, V. Rubtzov ${ }^{b}$, A. Sheifler ${ }^{\mathrm{a}}$, O. Suvorova ${ }^{\mathrm{a}}$, A. Shirokov ${ }^{\mathrm{c}}$, B. Shoibonov ${ }^{\mathrm{d}}$, Ch. Spiering ${ }^{\mathrm{e}}$, B. Tarashansky ${ }^{\mathrm{b}}$, R. Wischnewski $^{\mathrm{e}}$, A.Zagorodnikov ${ }^{\mathrm{b}}$, V. Zhukov ${ }^{\mathrm{a}}$, A. Yagunov ${ }^{\mathrm{b}}$, I.Yashin $^{\mathrm{c}}$

\author{
${ }^{a}$ Institute for Nuclear Research, Moscow, Russia \\ ${ }^{b}$ Applied Physics Institute of Irkutsk State University, Gagarin blvd. 20, Irkutsk 664003, Russia \\ ${ }^{c}$ Skobeltsyn Institute of Nuclear Physics MSU, Moscow, Russia \\ ${ }^{d}$ Joint Institute for Nuclear Research, Dubna, Russia \\ ${ }^{e}$ DESY, Zeuthen, Germany \\ ${ }^{f}$ Nizhni Novgorod State Technical University, Nizhni Novgorod, Russia \\ ${ }^{g}$ St.Petersburg State Marine University, St.Petersburg, Russia \\ ${ }^{h}$ Kurchatov Institute, Moscow, Russia
}

\begin{abstract}
The first stage Baikal Neutrino Telescope NT200 has been operating since 1998 and was upgraded to the 10 Mton detector NT200+ in 2005. The preparation towards a development of a km3-scale detector in Lake Baikal is currently a central activity point. As an important milestone a km3-prototype Cherenkov string, based on completely new technology, was installed in 2008 and has been successfully operating together with NT200+. It was upgraded in April 2009. Also, we review the status of high-energy acoustic neutrino detection activities in Lake Baikal.
\end{abstract}

Key words: Baikal neutrino telescopes, Acoustic neutrino detection

\section{Introduction}

Fifty years have passed since M. Markov proposed to build an array in the ocean to search for high energy astrophysical neutrinos [1]. Three large scale neutrino telescopes are currently under operation: AMANDA/IceCube [2] at the South Pole, ANTARES [3] in the Mediterranean, and the first of them, NT200 in lake Baikal [4]. The first stage underwater neutrino telescope configuration NT36 in lake Baikal was mounted in 1993. The full physics program was started in 1998 with the telescope configuration NT200 [5]. NT200 consists of 8 strings, each with 24 pairwise arranged optical modules (OM). Each OM contains a 37-cm diameter hybrid photodetector QUASAR-370, developed specially for this project [6]. The upgraded Baikal telescope NT200+ [7] was commissioned in April, 2005, and is made of a central part (the old, densely instrumented NT200 telescope) and three additional external strings. A review of the main physics results obtained with NT200 and NT200+ is presented for example in [8]. Good natural conditions (transparent fresh water, low light scattering, small water currents) and the possibility to use ice as a natural platform for detector deployment make the Baikal site very attractive for the creation of a large scale neutrino telescope with a volume of about a cubic kilometer. The Baikal collaboration follows since several years a R\&D program for a gigaton volume detector (GVD) in Lake Baikal. The most re- cent $\mathrm{km}^{3}$-milestones were the construction and installation of new technology prototype strings in 2008 and 2009. The basic goals of the prototype strings installation are investigation and in-situ test of basic elements of the future detector.

Probably the volume of a detector to search for super high energy cosmogeneous neutrinos should be much more than a cubic kilometer and far beyond the limits of reasonable size for an optical Cherenkov underwater array. Back in 1957, G. Askaryan showed that a high-energy particle cascade in water, besides the Cherenkov radiation, should also produce an acoustic signal [9]. The potential of the acoustic neutrino detection [10], [11] is based on the fact that the absorption length for acoustic waves with a frequency about $10-30 \mathrm{kHz}$ (the peak frequency of acoustic signals from a shower) in sea water is at least an order of magnitude larger than that of Cherenkov radiation, in the fresh Baikal water this ratio is even close to 100 [12]. Therefore, in principle, a deep-water acoustic detector for high-energy neutrinos can have a much smaller number of measuring channels than an optical Cherenkov detector with the same effective volume. But actually, the possibility of acoustic detection of high-energy neutrinos and the energy detection threshold are determined by the possibility of separating the neutrino-induced acoustic signal from ambient noise.

In this paper we review the present status of the R\&D of the km3-scale Baikal Cherenkov telescope and first results of our activities towards the development of an acoustic neutrino de- 
tection method.

\section{Gigaton Volume Detector in Lake Baikal}

Taking into account our previous experience with the NT200 design, a GVD will consist of strings of optical modules that will be grouped in clusters (see Fig. 1). This approach provides a relatively flexible structure, which allows to organize the power supply and data acquisition system of the underwater array in the optimal way and also to rearrange the main building blocks (clusters) of the GVD in order to adapt its configuration to requirements of new scientific goals if necessary. The objective of the GVD design optimization is to provide a maximum cascade detection volume and at the same time efficient registration of high energy muons. MC-optimization for the km3-detector design is going on. The present calculation was performed for an array with optical modules (OMs) containing photomultipliers R-7081HQE. Basic parameters for the optimization were $\mathrm{Z}$ - the vertical distance between OMs, R the distance between string and cluster center, and $\mathrm{H}$ - the distance between cluster centers. Coincidences of any neighboring $\mathrm{OM}$ on a string (thresholds 0.5 p.e. and 3 p.e.) were used as a trigger condition for this calculation. The compromise between cascade detection volume and muon effective area was obtained for the following values of the parameters: $\mathrm{H}=300 \mathrm{~m}$, $\mathrm{R}=60 \mathrm{~m}, \mathrm{Z}=15 \mathrm{~m}$. The preliminary design of GVD is presented in Figs. 1 and 2. It will contain a total of 2304 OMs, arranged on 96 strings with 24 OMs each, and an instrumented length of $345 \mathrm{~m}$. The effective area of the installation for muons above $10 \mathrm{TeV}$ with a reconstruction error of $0.5^{\circ}-1^{\circ}$ is $0.2-0.5 \mathrm{~km}^{2}$, and the effective volume for cascade events above $100 \mathrm{TeV}$ with a reconstruction error of $5^{\circ}$ is $0.2-0.7 \mathrm{~km}^{3}$.

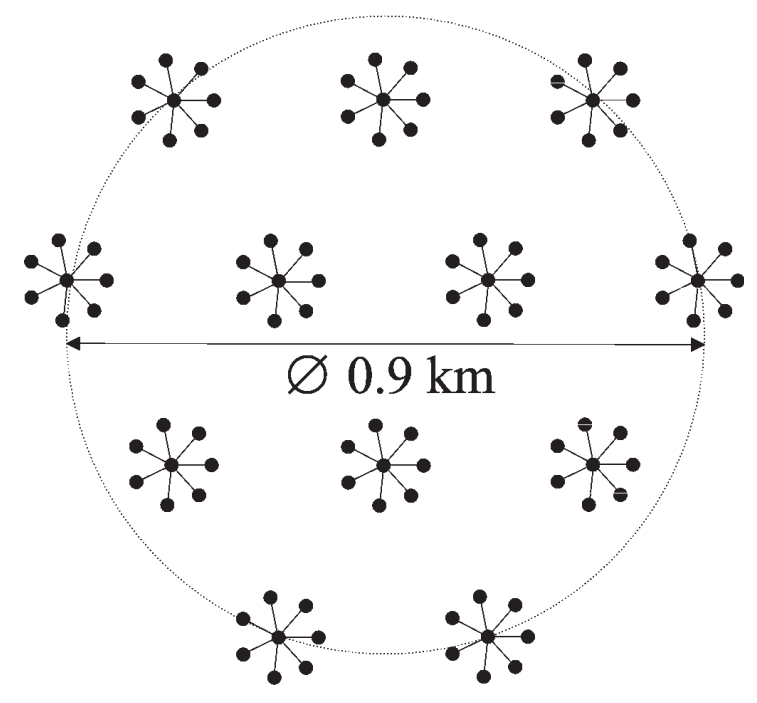

Figure 1: Top view of GVD: 96 strings are grouped in 12 Clusters.

\subsection{Data acquisition system of GVD}

The DAQ system of GVD is constructed from three basic building blocks: optical modules, sections of OMs (main de-

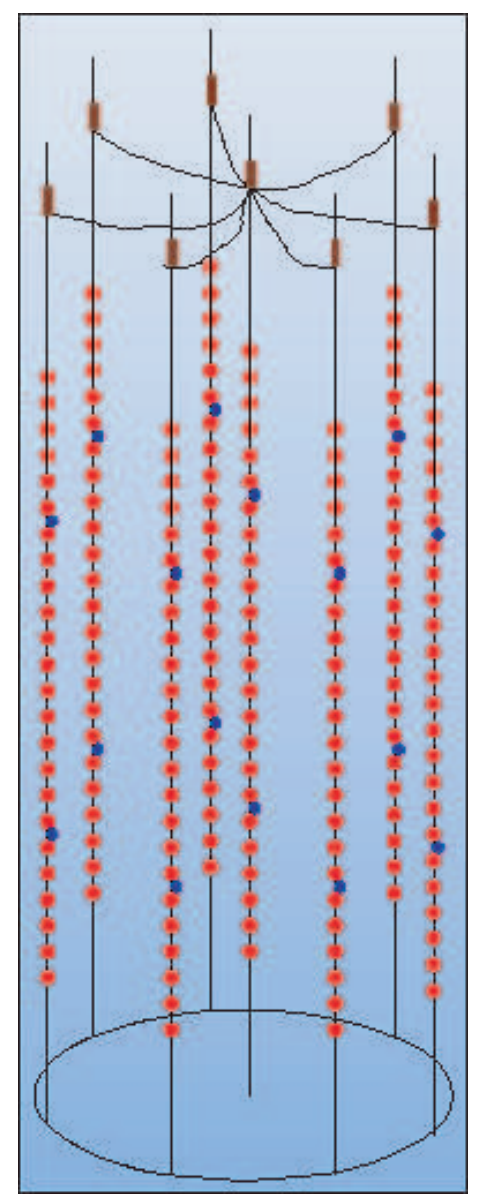

Figure 2: Schematic view of a cluster, made up of 8 strings with 192 OMs in total.

tection units) and clusters. The OM consists of a photomultiplier (PMT), a High Voltage unit (HV), two-channel preamplifier, and a controller. The block diagram of the OM electronics is presented in Fig. 3. The photomultiplier R-7081HQE was preliminary selected as light sensor for the OMs. This PMT has a hemispherical photo cathode with diameter 10" and quantum efficiency about $35 \%$. The OM controller is intended for $\mathrm{HV}$ regulation and monitoring, for permanent PM noise measurements, and for time and amplitude calibration of the measuring channel with two internal LED. Slow control data to and from the OMs are transferred via an underwater RS-485 bus. The OM electronics and the PMT are placed in a pressureresistant glass sphere with $42 \mathrm{~cm}$ diameter. Two connectors are foreseen in the glass sphere for communication. A high permittivity alloy cage surrounds the PMT, shielding it against the Earth's magnetic field. Optical modules of each string are grouped into two sections. The functional scheme of one section is presented in Fig. 4. The section is the basic detection unit of GVD. It consists of 12 OMs, the service module (SM) and the electronics unit (BEG) with analog-digital converters on the basis of FADCs. Analog signals from the OM are transmitted to the BEG through coaxial cables of 90 m length. BEG consists of three FADC boards (12 channels in total), an OM power controller and a VME controller. The VME controller 
provides trigger logic, data readout from FADC boards and connection via local Ethernet to the underwater PC on the cluster. The trigger is formed by a coincidence of any neighbouring OMs within a section (thresholds 0.5 and 3 p.e.). Every FADC board consists of four simultaneously digitizing FADCs. Every FADC has 12 bit resolution and samples at $200 \mathrm{MHz}$. The power control board is intended for the OM power supply. This module allows to switch power on/off for each optical module. Data from the BEG are transmitted via a Ethernet connection (10Mbit DSL-modem) to the underwater micro-PC of the cluster. Slow control of OMs is provided by BEG via a RS-485 underwater bus. Time and amplitude calibration of the section are provided by a LED flasher unit located in the service module (SM). The LED flasher provides all relative time shifts, and allows to monitor the single electron spectrum of all PMs. Basic elements of the LED flasher unit are two LED drivers and a LED controller. The LED controller has the same design as the OM controller, and provides the possibility of LED pulse amplitude and delay regulation independently for the two LEDs. Light pulses from the flasher are transmitted to each OM via individual plastic optical cables of calibrated length.

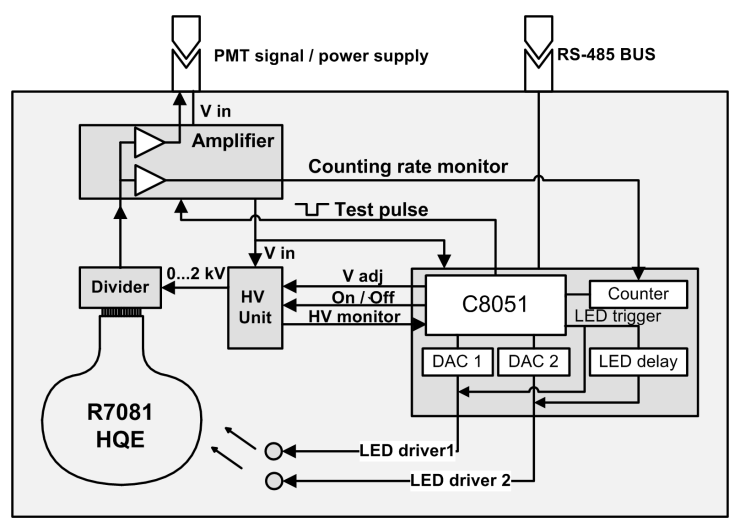

Figure 3: Functional scheme of the optical module electronics.

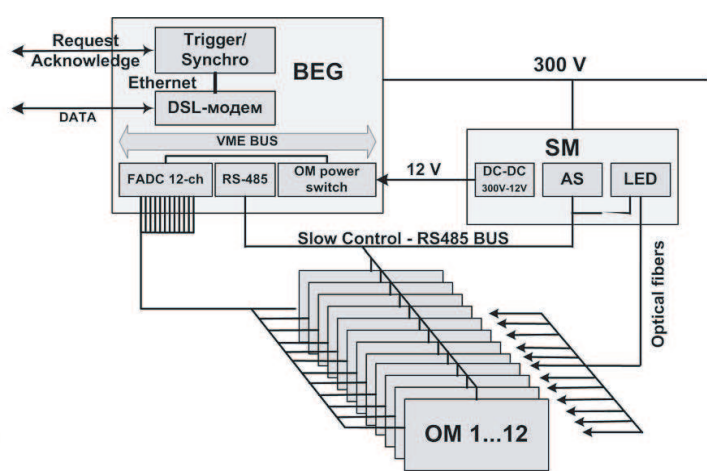

Figure 4: Functional scheme of the section of the optical modules: basic detection unit of GVD.

\subsection{Prototype string of GVD}

The first prototype of GVD electronics was installed in Lake Baikal in April 2008 [14]. It was a reduced size section with 6
OMs. This unit provided the possibility to study the basic elements of the future detector: new optical modules, the DAQ system of a section and the calibrating system. In April 2009 a prototype of the GVD string with two sections was installed as a part of the NT200+ detector. The objectives were studies of the basic DAQ/triggering approach for the km3-detector and investigation of timing accuracy in the synchronization of the sections. The prototype string consists of $12 \mathrm{OMs}$ with six photomultipliers R8055 (Hamamatsu, 13" photocathode) and six XP1807 (Photonis, 12" photocathode), two BEGs, a service module (SM) and a micro-PC unit. The distance between OMs along the string is $10 \mathrm{~m}$. The upper $10 \mathrm{OMs}$ have photomultipliers with downward looking photocathode, while the two bottom OMs contain PMs with upward looking photocathode. Data from the FADC units (BEG) are transmitted via a local Ethernet line to the underwater micro-PC unit that emulates the operation of the future cluster DAQ center. SM contains the string calibration system on the basis of LEDs and DC-DC converters for OM power supply.

\section{Acoustic neutrino detection activities}

Pressure $P$ of acoustic signal from a cascade of relativistic particles is described by a wave equation with a so called 'thermo-acoustic' source term:

$$
\rho \nabla\left(\frac{1}{\rho} \nabla P\right)-\frac{1}{c_{s}^{2}} \frac{\partial^{2} P}{\partial t^{2}}=-\frac{\alpha}{C_{p}} \frac{\partial^{2} q(\vec{r}, t)}{\partial t^{2}},
$$

where $q(\vec{r}, t)$ is the energy deposition density along the cascade. The acoustic emission depends on water characteristics: its density $\rho$, the adiabatic and non-dispersive speed of sound $c_{s}$, the thermal expansion coefficient $\alpha$ and the heat capacity $C_{p}$. These four parameters depend on the water temperature, its hydrostatic pressure and its salinity, the last factor is negligible in the case of lake Baikal. Taking into account our data for long-term temperature monitoring at the Neutrino telescope site, for lake Baikal it is possible to distinguish two depth intervals with qualitatively different temperature regimes. First, the near-surface zone $(0-150 \mathrm{~m})$, in which sizable seasonal variations of the water temperature are observed (from $0^{\circ} \mathrm{C}$ in winter up to $+18^{\circ}$ in summer just at the top). Second, the deep zone $(150 m-1370 m)$, in which the water temperature is very close to the temperature of maximum density (TMD) at depth 200-300m and slowly (more slowly then TMD) decreases with depth irrespective of season. As a result, at large depths the difference between TMD and Baikal water temperature amounts to $2^{\circ} \mathrm{C}$ (see also the measured depth-temperature profile shown in $[8])$.

Fig. 5 shows the time profile and frequency spectrum of an acoustic pulse at distance $1000 \mathrm{~m}$ from a neutrino-induced cascade with energy $10^{20} \mathrm{eV}$ at $1000 \mathrm{~m}$ depth in Baikal water, as simulated with our MC. To calculate the energy deposition density $q(\vec{r}, t)$ we used the CORSIKA program adapted to a water medium in [13]. Details of the calculations will be presented 
elsewhere. Because of the rather small value of the thermal expansion coefficient $\alpha$ in Baikal, the amplitude of the acoustic signals should be smaller then in the warm Mediterranean at the same cascade energy. Nevertheless, the possibility of acoustic detection of high energy neutrinos and the energy threshold of the array depend not only on signal amplitude but also on ambient noise from other sources, the efficiency of noise reduction and signal filters and also on absorption. To study noise from a wide range of sources in Lake Baikal and to test in practice techniques for acoustic noise reduction, signal filters and source position reconstruction, we have constructed a digital hydroacoustic module with four input channels, arranged on the corners of a regular tetrahedron, with edge length $1.5 \mathrm{~m}$ [15]. Long term monitoring of ambient noise with the instrument shows that the noise power spectral density decreases with a slope of about 5-6 dB/octave on average (see [16] for details). At any point of time the noise depends on the surface condition rather than on depth. At stationary and homogeneous meteorological conditions the integral noise power in the relevant frequency range $5-20 \mathrm{kHz}$ reaches levels as low as $\sim 5 \mathrm{mPa}$.
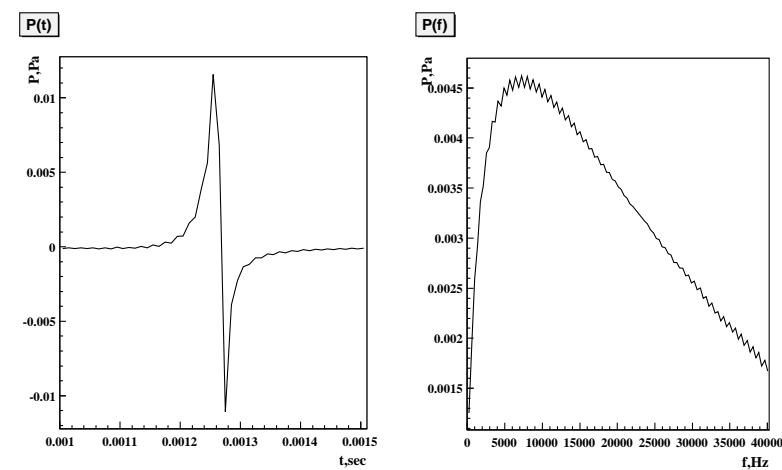

Figure 5: Form and spectra of an acoustic pulse at $1000 \mathrm{~m}$ distance from a neutrino-induced cascade shower of energy $10^{20} \mathrm{eV}$ occurring at $1000 \mathrm{~m}$ depth in Baikal water

The sensitivity of an acoustic particle detector depends to a large extent on the density and distribution of sources producing neutrino-like bipolar pulses and on the rate of such background signals. Reconstructing the position of a point source using one module with a small antenna of hydrophones becomes increasingly difficult. However, directional reconstruction is well possible using the arrival time differences for signals reaching the individual hydrophone. To test experimentally the angular resolution of the instrument and the reconstruction accuracy of the zenith and azimuth angles of incidence of acoustic signals, a hydrophone based calibrator set-up of bipolar signals was developed. The calibrator was installed underwater at a distance of $50 \mathrm{~m}$ from the antenna. About 5000 events were recorded and processed further by a correlation analysis procedure. Fig. 6 shows the distribution of acoustic signals versus zenith and azimuth angles during the measurement (calibration pulses are shown on the superimposed histogram). The RMS of the reconstructed angles are $\sigma_{\phi} \approx 1.5^{\circ}$ and $\sigma_{\theta} \approx 0.5^{\circ}$.

Taking into account that the main part of background sources (waves, wind, rain, ships, biological sources and so on) are con-

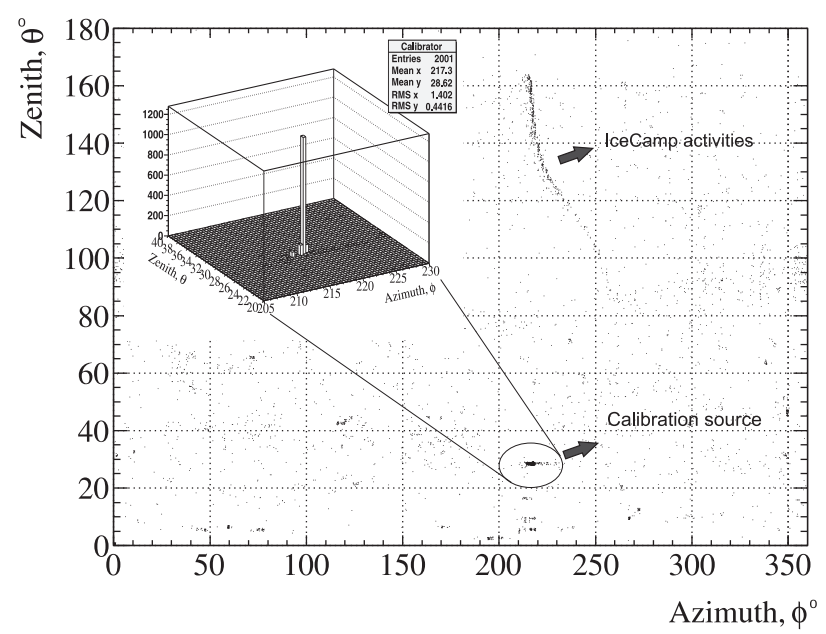

Figure 6: Distribution of acoustic signals versus zenith and azimuth angles including signals from calibration source.

centrated near the surface of a basin we chose the option of installing the acoustic module at a depth of $150 \mathrm{~m}$ at one of the moorings of NT200+ to acoustically watch the water body from top-down. Fig. 7 shows the distribution of detected acoustic signals versus zenith angles during the experiment. For all of the about 7000 reconstructed bipolar acoustic events we find arrival directions of zenith angle $<100^{\circ}$, i.e. they originate from sources located in the top zone of the Lake (note: pulses slightly below horizon with zenith angle $>90^{\circ}$ are expected due to refraction). Only a single event is reconstructed as occurring in the deep part of the lake. This event has a zenith angle of $174^{\circ}$ and is cleary isolated in fig. 7; the corresponding 4 hydrophone signals are shown in fig. 8.

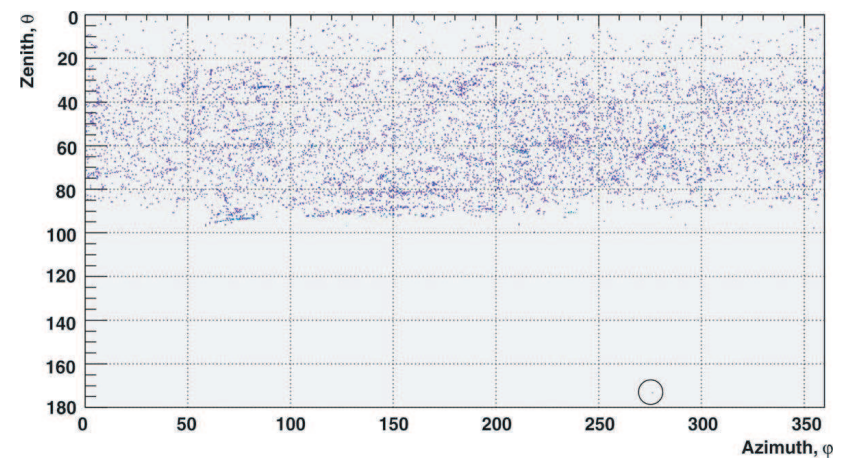

Figure 7: The distribution of reconstructed zenith versus azimuthal angle for events with bipolar pulses.

More information about position and properties of sources of neutrino-like pulses will be available with a grid of rather compact acoustic detectors arranged at shallow depths (100-200 m for Lake Baikal). An R\&D program to develop an acoustic string has been started in 2009. We plan a string that will consist of three acoustic modules with 4 hydrophones each. The modules are equipped with PICO-ATOM industrial PCs, which communicate with each other via a dual-channel 100/1000 Mbit 

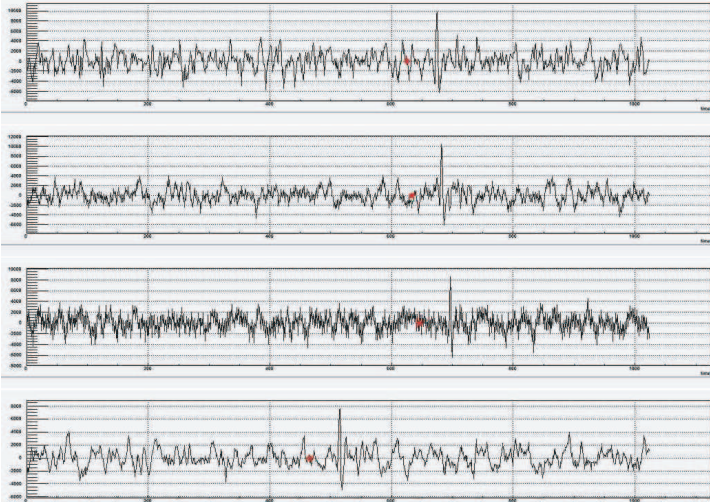

Figure 8: Acoustic event reconstructed as upward-neutrino like event (see fig. 7): raw pulse traces (channels 1-4).

Ethernet line. The top module on the string will have an additional DSL connection to the NT200+ underwater network. Each acoustic module will be composed of directive waveguide sensors, which possess high uniform sensitivity within a 90 degrees wide cone, offering better noise conditions to search for near-horizontal UHE neutrinos in deep water layers of the lake. For acoustic signal digitization we examine professional Infrasonic QUARTET 4x4 channel audio cards with 24-bit/192kHz sigma delta ADCs.

\section{Support}

This work was supported in part by the Russian Ministry of Education and Science (GK 02.740.11.0018, P1242, P2504, zadanie RNP 2.2.1.1/1483, RNP 2.1.1/1539, RNP 2.2.1.1/5901, REC "BAIKAL"), by the German Ministry of Education and Research, by the Russian Found for Basic Research (grants 0802-00432-a, 09-02-10012, 07-02-00791, 08-02-00198, 09-0210001, 09-02-00623, 09-02-12295), by the grant of the President of Russia NSh-321.2008-2, , and by NATO Grant NIG9811707(2005).

\section{References}

[1] Markov M.A. On high energy neutrino physics // Proc. 1960 Annual Int. Conf. on High Energy Phys., Rochester. 1960 P.578.

[2] A. Achterberg at al., Astropart. Phys. 26 (2006) 155.

[3] J. Carr [ANTARES Collaboration], Nucl. Instr. and Meth. A588 (2008) 80.

[4] V. Aynutdinov et al., Astropart. Phys. 25 (2006) 140.

[5] I.Belolaptikov et al., Astropart. Phys. 7 (1997) 263.

[6] R.Bagduev et al., Nucl. Instr. and Meth. A420, (1999) 138.

[7] V. Aynutdinov et al., Nucl. Instr. and Meth. A588 (2008) 99.

[8] V.Aynutdinov et al., Nucl. Instr. and Meth. A 602 (2009) 14.

[9] G. A. Askaryan, Atomnaya Energiya. 3 (1957) 152.

[10] G. A. Askaryan et al Nucl. Instr. and Meth. 164 (1979) 267.

[11] J. G. Learned, Phys. Rev. D 19 (1979) 3293.

[12] C. S. Clay and H. Medwin, Acoustical Oceanography (Wiley, New York), 1977.

[13] S. Bevan et al. Astropart.Phys.28 (2007) 366.

[14] V. Aynutdinov et al., Nucl. Instr. and Meth. A602 (2009) 227.
[15] V. Aynutdinov et al., Nucl. Instr. and Meth. A 604 (2009) 130.

[16] V. Aynutdinov et al., Acoust. Phys. 52 (2006) 495. 